\title{
ON NAKAYAMA'S EXTENSION OF THE $x^{n(x)}$ THEOREMS
}

\section{ALEX ROSENBERG AND DANIEL ZELINSKY}

In [6] Nakayama proved that if $A$ is a division ring with center $Z$ such that every element $a$ of $A$ satisfies

$$
f(a)=a^{n_{1}(a)} \alpha_{1}+\cdots+a^{n_{r}(a)} \alpha_{r} \in Z
$$

where the $\alpha_{i}$ are $r$ fixed nonzero elements of $Z$, and $0<n_{1}(a)$ $<n_{i}(a)(i=2, \cdots, r)$, then $A=Z$. In $[3$, Theorem $11 ; 5 ; 2 ; 1]$ specialized forms of (1) (e.g. $a^{n(a)} \in Z, a^{n(a)}-a \in Z$ ) are shown to imply commutativity at least for semi-simple rings. It is natural therefore to seek an extension of Nakayama's result to semi-simple rings. Since a semi-simple ring is a subdirect sum of primitive rings and (1) is preserved under homomorphism we first study primitive rings satisfying (1).

If $A$ is such a ring it may be identified with a dense ring of linear transformations on a vector space $\mathfrak{M}$ over a division ring $D$. Since $D$ is the ring of endomorphisms of $\mathfrak{M}$ that commute with $A, Z$ is in $D$ and in fact in the center of $D$. We may assume that there are at least two independent vectors $x, y$ in $\mathfrak{M}$. If $\lambda \in D$ there is an element $a$ in $A$ such that $x a=0, y a=y \lambda$. Now $y a^{n}=y \lambda^{n}$ so that if $f(a) \in Z$ is the relation of type (1) that $a$ satisfies, we have $x f(a)=0, y f(a)=y f(\lambda)$. Since $f(a) \in Z \subset D$ this makes $f(a)=0$ so that $y f(\lambda)=0, f(\lambda)=0$, and by Nakayama's result $D=Z$. Thus the center of a primitive ring satisfying (1) is a field.

Moreover, we have proved that each element in this field satisfies a polynomial equation of the form $f(a)=0$ with $f$ as in (1). Let $P$ be the prime subfield of $Z$ and $Q$ be the field obtained by adjoining to $P$ a maximal, algebraically independent set from among $\alpha_{1}, \cdots, \alpha_{r}$. Assuming that $Z$ is not absolutely algebraic ${ }^{1}$ of prime characteristic, Lemmas 1 and 2 of [6] show that $Z$ is purely inseparable over $Q$ or $Z=Q$. In the former case $f(a)^{p^{k}}=0$ is an equation with coefficients in the rational function field $Q$; thus in either case every nonzero rational function $\lambda$ would satisfy an equation

$$
\beta_{1}+\beta_{2} \lambda^{n_{2}(\lambda)}+\cdots+\beta_{r} \lambda^{n_{r}(\lambda)}=0
$$

with $n_{i}(\lambda) \geqq 1$ and the $\beta_{i}$ fixed polynomials. [If $Q=P=$ the rationals we take the $\beta_{i}$ to be integers.] However the ring of polynomials in several variables [the ring of integers] is a unique factorization do-

Presented to the Society, June 20, 1953; received by the editors May 27, 1953.

1 Algebraic over its prime subfield. 
main and so the numerator of $\lambda$ would have to divide $\beta_{1}$ for every $\lambda$ in $Q$, which is impossible. Hence $Z$ is an absolutely algebraic field of characteristic $p$.

Thus a primitive ring satisfying (1) is an algebraic algebra over an absolutely algebraic field of characteristic $p$; this automatically makes it an algebraic algebra over $G F(p)$. Conversely, every algebraic algebra over $G F(p)$ satisfies a very special form of (1). For if $a$ is an element of $A$, there is a polynomial $f$ over $G F(p)$ such that $f(a)$ $=0$. The splitting field of $f$ is a finite field, so that for some $q=p^{s}$, each linear factor of $f$ divides $x^{q}-x$. If $m$ is a power of $p$ larger than the multiplicity of each root of $f$, then $f$ divides $x^{m q}-x^{m}$. Hence $a$ satisfies $a^{m q}-a^{m}=0$. We have

TheOREM 1. A noncommutative primitive ring satisfies (1) if and only if it is an algebraic algebra over its center ${ }^{2}$ and this center is an absolutely algebraic field of prime characteristic. In this case, the condition (1) implies $a^{n(a)}-a^{m(a)}=0,0<n(a)<m(a)$.

Next, if $A$ is a semi-simple ring satisfying (1) with $\alpha_{1}$ in no primitive ideal, it is clear that $A$ will be a subdirect sum of rings $C$ and $A_{p}$, where $C$ is a commutative semi-simple ring and each $A_{p}$ is a subdirect sum of noncommutative primitive algebraic algebras over $G F(p)$. However, not every subdirect sum of this last form will satisfy (1): A complete direct sum of an infinite number of copies of the ring of $2 \times 2$ matrices over the algebraic closure of $G F(p)$ does not satisfy any condition of type (1). We can also see that (1) cannot always be replaced by $a^{n(a)}-a^{m(a)}=0$ in such an $A_{p}$. To obtain an example, let $Z_{i}$ be the algebraic closure of $G F(p), A_{i}$ the ring of all $2 \times 2$ matrices over $Z_{i}$, and $A$ the local direct sum ${ }^{3}$ of the $A_{i}$ with respect to the $Z_{i}$. Then for each $a$ in $A, a^{n(a)}-a^{m(a)}$ is in the center of $A$, but $A$ is clearly not algebraic over any field. In general we do not know whether (1) can always be replaced by the condition $a^{n(a)}-a^{m(a)}$ in the center. However, if the $n_{i}(a)$ are bounded we can settle the question; in fact:

THEOREM 2. Let $A$ be a semi-simple ring satisfying (1) with $\alpha_{1}$ in no primitive ideal and with bounded $n_{i}(a)$. Then $A$ is a subdirect sum of $a$ commutative ring and total matrix algebras with a bounded number of

${ }^{2}$ We remark that Theorem 1 and its proof remain true verbatim if we replace the center of $A$ throughout by the centroid of $A$-the ring of endomorphisms of the additive group of $A$ which commute with the left and right multiplications [4, p. 236].

${ }^{3}$ Each element of $A$ is an element of the Cartesian product of the $A_{i}$ having its $i$ th component in $Z_{i}$ for all but a finite number of $i$ 's. 
elements. Furthermore, $A$ satisfies the condition $a^{n}-a^{m} \in Z$ with $n$ and $m$ fixed, unequal positive integers.

Proof. Let $k$ be a bound for $n_{i}(a)$ and $r$ be as in (1). We show that every noncommutative primitive image $B$ of $A$ has $\leqq l$ elements, where $l$ is a function of $k$ and $r$ alone. By our previous arguments each element $\eta$ of the center $Y$ of $B$ satisfies an equation $f(\eta)=0$ with $f$ as in (1). Since the degree of $f$ is $\leqq k$ there are at most $k^{r}$ such polynomials. Let $g$ be their product; its degree is at most $s=k^{r+1}$, and every element of $Y$ is a root of $g$. Hence $Y$ is a field with at most $s$ elements. Now $B$ is a primitive algebraic algebra of degree $\leqq k$ over $Y$ and so by [3, Theorems 5 and 8 ] it is a total matrix algebra of degree $\leqq k$ over $Y$. Thus $B$ has at most $l=s^{k^{2}}$ elements.

Hence $B$ is an algebraic algebra over $G F(p)$ of dimension $<l$. Therefore every element $b$ of $B$ satisfies a polynomial equation of degree $<l$ and with coefficients in $G F(p)$. The remarks immediately preceding Theorem 1 then show that there exist positive integers $u, v$ depending only on $l$ and $p$ such that $b^{u}-b^{v}=0$ for all $b$ in $B$. If we write $A$ as a subdirect sum of the rings $C$ and $A_{p}$ as before, each $A_{p}$ will satisfy a polynomial identity $a^{u}-a^{v}=0$. Since the noncommutative primitive summands are of bounded size, at most a finite number of distinct $A_{p}$ 's can occur. To complete the proof it suffices to choose positive, unequal integers $m, n$ such that $x^{n}-x^{m}$ is divisible by each of the finite number of polynomials $x^{u}-x^{v}$.

\section{REFERENCES}

1. I. N. Herstein, A generalization of a theorem of Jacobson III, Amer. J. Math. vol. 75 (1953) pp. 105-111. 240.

2. M. Ikeda, On a theorem of Kaplansky, Osaka Math. J. vol. 4 (1952) pp. 235-

3. N. Jacobson, Structure theory for algebraic algebras of bounded degree, Ann. of Math. vol. 46 (1945) pp. 695-707.

4. N. Jacobson, Structure theory of simple rings without finiteness assumptions, Trans. Amer. Math. Soc. vol. 57 (1945) pp. 228-245.

5. I. Kaplansky, $A$ theorem on division rings, Canadian Journal of Mathematics vol. 3 (1951) pp. 290-292.

6. T. Nakayama, On the commutativity of certain division rings, Canadian Journal of Mathematics vol. 5 (1953) pp. 242-244.

NORTHWESTERN UNIVERSITY 